\title{
DISK STRUCTURE IN U GEMINORUM
}

\author{
J. Madej \\ Warsaw University observatory \\ B. Paczyński \\ N. Copernicus Astronomical Center \\ Polish Academy of Sciences \\ Poland
}

\begin{abstract}
ABST RACT
A hot spot at the outer rim of the accretion disk dominates the light of $U$ Geminorum at minimum light. We take this as evidence that there is no accretion from the disk onto the white dwarf between the eruptions, and we assume there is no viscosity in the disk at that time. The hot spot is produced by dissipation of the radial component of velocity of stream falling from the inner Lagrangian point. Angular momentum per unit massis smaller in the stream than it is in the outer parts of the disk. This leads to angular momentum redistribution in the outer part of the disk. The difference of tangential velocity between the stream and the disk is dissipated in few orbital periods. These processes make the outer parts of the disk look like a torus. We calculated the structure of the torus in U Geminorum between the eruptions and we obtained the following parameters: mass of the torus: $10^{-9}-10^{-8} \mathrm{~m}$ o (assumed), its optical thickness: $10^{6}$, the large radius (i.e. the radius of the disk): $0.5 \mathrm{R}_{0}$ (assumed), the small radius (i.e. the half thickness of the outer parts of the disk): $0.05 R_{\odot}$. Conditions at the surface of the torus are similax as on the solar surface.
\end{abstract}


A hot spot is a very prominent feature in dwarf novae at minimum light. It is believed to be formed at the outer rim of the accretion disk by the stream of gas flowing from the inner Lagrangian point (Smak 1871). No model of the hot spot exists in the literature. One of the problems is lack of knowledge of the structure of outer rim of the disk. In this paper we present a simple model of the rim. In future we intend to use this model for a study of the hot spot structure.

We assume that the following interpretation of observations of $\mathrm{U}$ Geminorum is correct. Mass is transferred from the red dwarf filling its Poche lobe onto the outer rim of the disk rotating around the white dwarf. The size of the disk is about equal to the size of the largest simple periodic particle orbit which does not intersect other orbits (Smak 1976, Paczynski 1977). Therefore, angular momentum per unit mass is higher at the outer rim of the disk than in the stream of gas flowing from the inner Lagrangian point. The hot spot is produced by dissipation of the radial component of velocity of the stream. The difference of tangential velocity between the stream and the disk is dissipated into heat in few orbital periods of the outer rim. There is no accretion within the disk and onto the white dwarf at minimum light (Osaki 1974). The radius of the outer rim of the disk decreases with time as relatively low angular momentum matter is deposited by the stream. At a certain moment viscosity within the disk increases and $a$ lot of heat is produced and radiated away. This is an outburst (Osaki 1974). At the same time viscosity leads to accretion of some matter onto the white dwarf, while the outer parts of the disk are pushed out. We believe, that tidal torques on the outer parts of accretion disk (Papaloizou and Pringle 1977) are so efficient that the outer rim cannot expand beyond the largest nonintersecting particle orbit. The excess of angular momentum is carried from the disk back to the orbital motion by the tidal torques. After the outburst viscosity in the disk vanishes and the accretion of matter from the stream onto the outer rim of the disk leads to the decrease of the disk size. We use this process to explain the well observed variations of the disk size with time in U Geminorum (Smak 1971).

The aim of this contribution is not to discuss the validity of the scheme presented above, but to model the structure of the outer region of the disk assuming this scheme to be correct. 
We assume there is no viscosity in the disk at minimum light. For simplicity we assume that the streamlines in the disk are circular, even at the outer rim. That means we ignore the influence of the secondary and the impact of the stream. We assume that the deposition of low angular momentum matter at the outer rim leads to dynamical instability which tends to keep the angular momentum per unit mass constant with radius within the outermost regions of the disk. Dissipation of the difference in tangential velocities between the stream and the outermost region of the disk leads to the heating of this region. We assume there is a thermal balance between this heating and the radiative losses from the surface of the outermost region of the disk leads to the heating of this region. We assume there is a thermal balance between this heating and the radiative losses from the surface of the outermost region of the disk which rotates with constant angular momentum per unit mass.

Is the temperature is finite, the pressure is also finite, though small. The small pressure gradient makes it possible to maintain an equilibrium in the region of constant angular momentum per unit mass. If pressure was vanishingly small than all the mass accreted onto the outer rim would form just a single streamline. As pressure is finite this matter will flow along a tube of a finite crossection. Taking thermal balance into account we shall find the size of the tube. The tube has to be large enough to be able to radiate away the heat generated within the tube. Let us emphasize that the heat is generated by dissipating differences in specific angular momentum. In our model matter within the tube rotates with angular velocity varying with radius, but shear does not lead to heat dissipation as we assume that viscosity is vanishingly small.

Let us consider, for simplicity, a barytropic equation of state, $P=P(\rho)$, and axisymmetric rotation of the disk. The combined potential due to gravitational field of the white dwarf and that due to rotation may be written as:

$$
u=\frac{G m}{r}+\int \Omega^{2} R d R \text {, }
$$

where $m$ is the white dwarf mass, $r$ is the distance from the white dwarf center, $R$ is the distance from the rotation axis and $\Omega$ is the angular velocity of rotation. He consider now small region where angular momentum per unit mass is constant and given as: 


$$
j=\Omega R^{2}=j_{0}=\left(G m R_{0}\right)^{1 / 2},
$$

where $R_{0}$ is the radius at which the angular momentum on a Keplerian circular orbit is equal to $j_{0}$. Combining eqs. (1) and (2) we find:

$$
u=G m\left(\frac{1}{r}-\frac{R_{0}}{2 R^{2}}\right)+\text { const }
$$

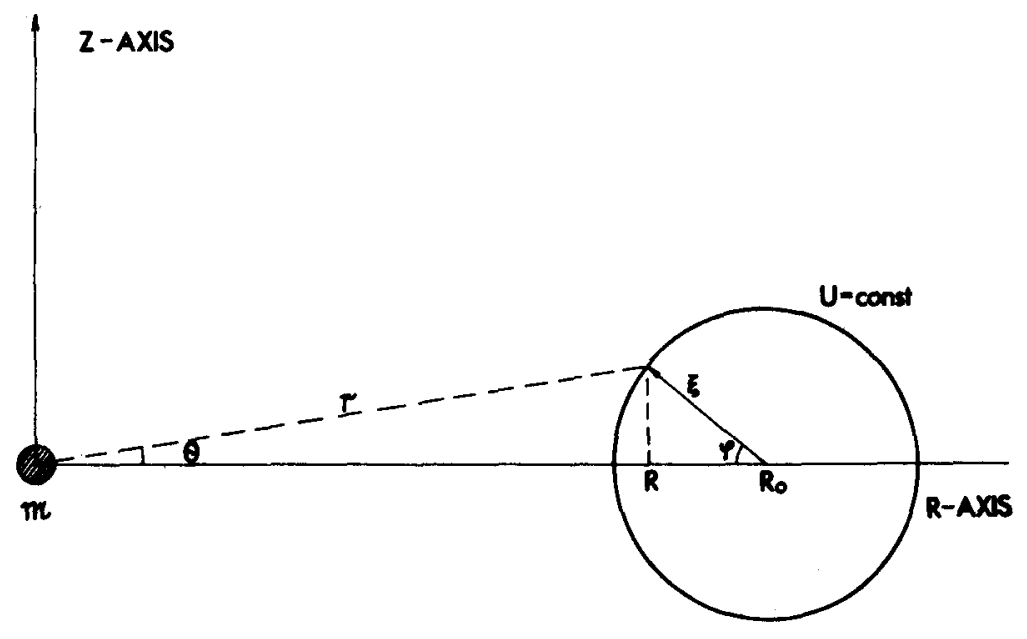

Fig. 1. Geometry of the problem. $M$ is the white dwarf mass. $R$ is a large radius of the torus. $\xi$ is a small radius of the torus.

In a small region near $R_{0}$ we may expand eq. (3) in a power series of $\xi$, the distance from the equatorial streamline with radius $R_{0}$ (cf. Fig. 1). Neglecting higher order terms we find: 


$$
U=u_{0}-\frac{G m}{2 R_{0}}\left(\frac{\xi}{R_{0}}\right)^{2},
$$

where $U_{0}$ is the potential at $R_{0}$. As we see an equipotential surface close to $R_{0}$ is a torus with a large radius $R_{0}$, and a small radius $\xi$. In the barytropic case this would also be a surface of equal pressure.

We have no a priori reason to expect that in the disk there is a barytropic relation between pressure and density. We assume this for simplicity. Therefore, in our model, at the outer region of the disk every surface of equal density, pressure and temperature is a torus. We expect that $\xi \ll R_{0}$, and a torus may be locally represented with a cylinder. Selfgravity of the cylinder (or torus) is neglected. Nevertheless, effective gravitational acceleration is directed towards the axis of the cylinder, and it is given as:

$$
g=\frac{d U}{d \xi}=-\frac{G m}{R_{0}^{3}} \xi
$$

Structure of the torus may be described with the stellar structure equations adapted to the cylindrical symmetry. These may be written as:

$$
\begin{aligned}
& \frac{d P}{d \xi}=+g \rho=-\frac{G m}{R_{0}^{3}} \xi \rho \\
& \frac{d m_{\xi}}{d \xi}=4 \pi^{2} R_{0} \xi \rho, \\
& \frac{d T}{d \xi}=\nabla \frac{T}{P} \frac{d P}{d \xi}, \quad \nabla=\min \left(\nabla_{\text {rad }}, \nabla_{\text {conv }}\right)
\end{aligned}
$$

where $m_{\xi}$ is the mass contained within the torus with radii $R_{0}$ and $\xi$. The radiative and convective gradients may be calculated like in a spherical case if we know the heat flux, $F_{\xi}$. Unfortunately, we know nothing about the distribution of heat sources, and we had to assume that:

$$
F_{\xi}=F_{0}\left(\frac{\xi}{\xi_{0}}\right)^{a}
$$


where $F_{O}$ is the surface heat flux (i.e. the surface brightness) at the torus with a surface radius $\xi_{0}$. The exponent a was treated as a free parameter. The stellar envelope program (Paczynski 1969) was adapted to the case of cylindrical symmetry and used to integrate eqs. (6) with the mixing length equal to one pressure scale helght. Normal chemical composition $(X=0.7, Y=0.27, \mathrm{Z}=0.03)$ was adopted. As we intended to model the case of $U$ Geminorum, we adopted:

$$
\begin{aligned}
& m=m_{0}, \\
& R_{0}=0.5 R_{0},
\end{aligned}
$$

following smak (19.76). The surface radius of the torus, $\xi_{0}$, and the total heat generation rate within the torus, $L$ (i.e. the luminosity of the torus), were used as free parameters. We have; of course a relation:

$$
F_{0}=\frac{L}{4 \pi^{2} R_{0} \xi_{0}}
$$

Mass of the torus was found as a result of integrations of the structure equations $(6)$.

Preliminary computations with the parameter a (cf. eq. 7) taken as 0,1 , and 2 demonstrated that the outer parts of the torus were convective, while the inner parts were radiative in some models. Thenever the interior tended to be radiative, we nevertheless assumed adiabatic temperature gradient, which effectively corresponds to modification at the distribution of heat sources (cf. eq. 7). Our reasoning is as follows. Accretion of mass with deficit of angular momentum must lead to dynamical instabilities and mixing of matter within the torus. In the interior the thermal timescale is long compared to the dynamical time scale and mixing driven by the gradient of specific angular momentum may lead to adiabatic temperature distribution.

The results of model computations were practically independent on the choice of $a$. Therefore most models were calculated with $a=1$. Alltogether a few dozens of models were calculated with varlous values of luminosity $L$, and thickness $\xi_{0}$ of the torus. The variation of torus, mass with its luminosity $L$, and small radius $\xi_{0}$ is shown in Fig. 2. The results interpolated to the round values of torus mass are given in Table $1 . T_{c}$ and $\rho_{c}$ are the temperature and density on 
the circular "axis" of the torus, $\tau$ is the optical depth from $\xi=0$ to $\xi=\xi_{0}, \mathrm{~T}_{\mathrm{e}}$ and $\mathrm{g}_{0}$ are the effective temperature and gravity at the torus surface.

Table 1

Parameters for some torus models with $L=0.1 \mathrm{~L}_{0}$ (cgs units)

\begin{tabular}{|c|l|l|l|}
\hline$m_{t} / m_{0}$ & $10^{-9}$ & $10^{-8}$ & $10^{-7}$ \\
\hline$\xi_{0} / R_{0}$ & 0.044 & 0.050 & 0.059 \\
$T_{c}$ & $5.110^{4}$ & $5.610^{4}$ & $6.610^{4}$ \\
$\rho_{c}$ & $1.010^{-6}$ & $810^{-6}$ & $510^{-5}$ \\
$\tau$ & $510^{+5}$ & $210^{+7}$ & $110^{+9}$ \\
$T_{e}$ & 6320 & 6120 & 5870 \\
$g_{0}$ & $9.60_{10^{3}}$ & $1.0910^{4}$ & $1.2910^{4}$ \\
\hline
\end{tabular}

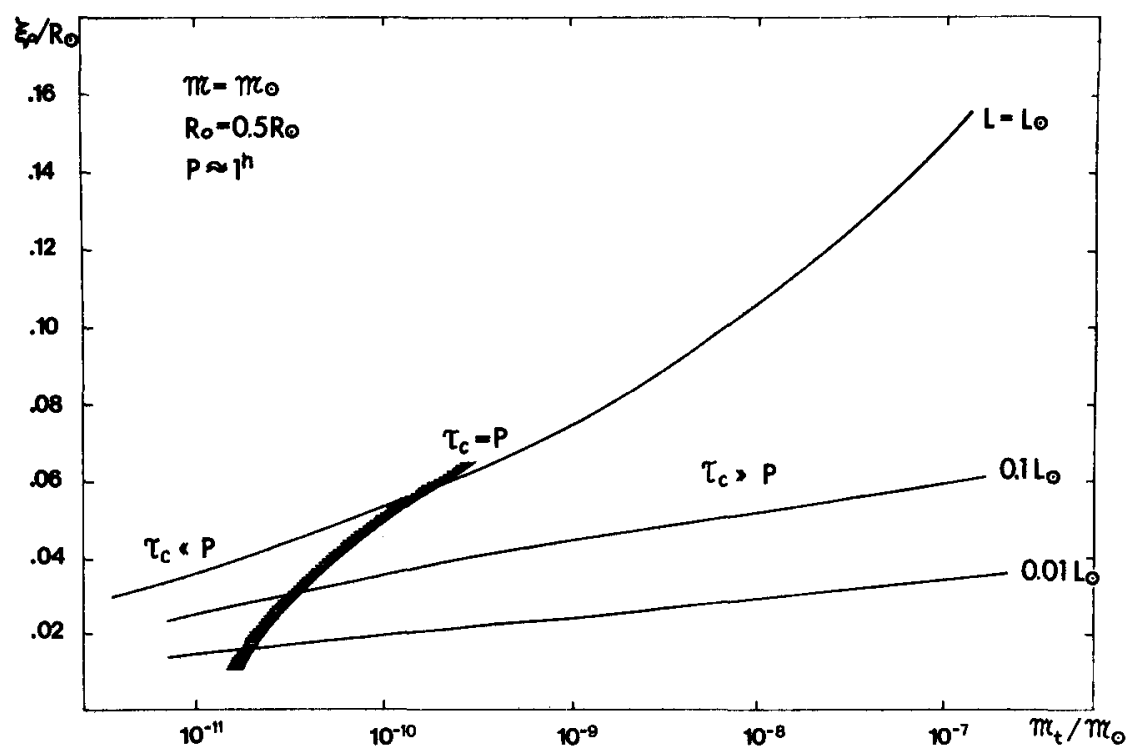

Fig. 2. Variation of the torus radius $\xi_{0}$ as a function of torus mass and luminosity. Line of thermal time scale equal to rotational period of the disk is indicated. 


\section{Conclusions}

The likely accretion rate in U Geminorum is in the range $10^{-9}-$ $10^{-8} \mathrm{~m}_{\odot} / \mathrm{Y}$ (smak 1976). This corresponds to the torus luminosity in the range $10^{-2}-10^{-1} \mathrm{~L}_{\odot}$. The torus mass should be around $10^{-9}$ $10^{-3} m_{\odot}$ as the torus contain not only the freshly accreted matter, but also swept up matter from the outer parts of the disk. Our models indicate that the small radius of the torus is close to $10 \%$ of its large radius. The thermal time scale is many days, and is much longer than the dynamical time scale (i.e. rotational period of the disk), which is about one hour. It seems reasonable to expect that because of its thermal inertia the torus should be uniformly bright along its circumference. The torus is so optically thick that it may be considered to be a toroidal star. Effective temperature and gravity at the surface of the torus are not very different from those of the sun.

It is a great pleasure to acknowledge that all the computations on which this paper is based were done with the PDP 11/45 computer donated to the Copernicus Astronomical Center by the Us National Academy of sciences following the initiative and through the action of Dr. R.C.O'Dell and Mr. A.M.Baer.

\section{$\underline{\text { References }}$}

Osaki, Y.

Paczynski $\mathrm{B} . \ldots \ldots \ldots \ldots \ldots \ldots$

Paczynski, B. ...............

Papaloizou,J. and Pringle,J.E. .

Smak, J .

Smak, J.
1974, P.A.S.J., 31, 429.

1969, Acta Astron., 19, 1.

1977, Ap.J., in press.

1977, preprint: "Tidal torques on accretion discs in close binary systems".

1971, Acta Astron., 21, 15.

1976, Acta Astron., $\underline{26}, 277$.

D I SCUSS I ON Of paper by MADEJ and PACZYKSSKI:

PRINGLE: 1. I object to your statement that it is a well-known observational fact that there is no accretion onto the white dwarf $U$ Gem during quiescence. This depends on how the observations are interpreted and it is my opinion that the full effect of photometric corrections has been overlooked in this context. I think it is fair to say that the question of whether or not accretion continues during quiescence has yet to be resolved.

2. The radius you assumed for your torus is larger than you would get from giving the matter in the torus the specific angular 
momentum in the initially transferred material. The matter in the torus seems therefore to have acquired some specific angular momentum from somewhere. Could you explain where this comes from?

MADEJ: 1. The statement that there is no accretion within the disk and onto the white dwarf at the time between eruptions is assumed following Osaki (1974). I agree with you that at the present time it is difficult to verify that assumption (or assumption of constant specific angular momentum in the outer parts of accretion disk) without any doubts. Therefore, the models presented above would wait for comparison with future observations only, not with speculative and uncertain theoretical considerations.

2. We have assumed the maximal disk radius $R_{0}$ following available observations (Smak 1976). Disk models in U Gem with smaller $R_{0}$ were not computed until now. As regards your question: at the time of eruptions caused by rapid increase of accretion rate unto the whyte dwarf, the accreted matter may transfer part of its angular momentum to the outer parts of the disk and consequently, increase the disk radius at the time of outburst. Unfortunately, no mechanism responsible for the above statement can be proposed now.

DZIEMBOWSKI: In answer to Dr. Pringle's second question, there is an angular momentum exchange between the outer and the inner layers of the disk 1mplicit in Madej and Paczynski's model. It is due to turbulence resulting from the rotational instability.

SMAK: A comment on accretion and viscosity not operating between outbursts; there appears to be no observational evidence for any significant accretion (and therefore viscosity) between the outbursts. To the contrary, we have Lspot 2 Laisk (at least in the visual region) and that is taken as an argument that there is No accretion. Of course, the problem of bolometric corrections still leaves some uncertainty there. 